\title{
Espessamento do tendão muscular na oftalmopatia de Graves
}

\section{Muscle tendon enlargment in Graves'ophthalmopathy}

Elvira Barbosa Abreu ${ }^{1}$

\begin{abstract}
ResUmo
Objetivo: Estudar a frequência do espessamento do tendão muscular em pacientes com oftalmopatia de Graves buscando estabelecer correlações com as diferentes formas clínicas da doença. A diversidade clínica e laboratorial na oftalmopatia de Graves pode levar à confusão quanto ao diagnóstico, conduta e prognóstico. Os achados radiológicos variam desde o aumento isolado do tecido adiposo até o espessamento da musculatura extraocular, caracteristicamente poupando os tendões. Em 2004,no entanto, Ben Simon descreveu o espessamento do tendão muscular na oftalmopatia de Graves. Métodos: Foram avaliados 20 pacientes, de ambos os sexos, com idades entre 20 e 80 anos, com formas clínicas designadas como :forma benigna (retração palpebral),forma intermediária (diplopia na posição primária do olhar) e forma maligna ou infiltrativa (sinais de comprometimento do nervo óptico).Todos os pacientes foram submetidos à tomografias computadorizadas de órbita. Os pacientes estavam eutiroideanos, há pelo menos um ano. Os padrões tomográficos foram estudados e divididos em dois grupos: com e sem espessamento dos tendões da musculatura extraocular. Resultados: Uma relação estatisticamente significativa entre espessamento do tendão e a forma clínica intermediária foi encontrada $(\mathrm{p}<0,012)$. Conclusão: $\mathrm{O}$ espessamento do tendão extraocular, encontrado em $30 \%$ dos pacientes com oftalmopatia de Graves, relaciona-se positivamente com a forma intermediária da doença, caracterizada pela presença de diplopia e que constitui um achado de grande valor clínico.

Descritores: Oftalmopatia de Graves/diagnóstico; Tomografia computadorizada por raios x; Exoftalmos; Órbita
\end{abstract}

${ }^{1}$ Oftalmologista do Instituto Penido Burnier - Campinas (SP), Brasil.

Instituto Penido Burnier - Campinas (SP), Brasil.

Os autores declaram inexistir conflitos de interesse

Recebido para publicação em: 8/7/2010 - Aceito para publicação em 29/8/2011

Rev Bras Oftalmol. 2011; 70 (6): 378-83 


\begin{abstract}
Purpose: The aim is therefore to study the frequency of tendon enlargment in Graves' ophthalmopathy, seeking to estabilish its clinical correlations. Clinical and laboratory diversity in Graves' Ophthalmopathy sometimes may mislead its diagnosis. Radiological findings are more reliable for the diagnosis of Graves' Ophthalmopathy. Since then, a number of patterns have been described. Extraocular muscle involvement in this pathology is considered as always sparing the tendons. In 2004, Ben Simon described extraocular muscles tendon enlargment in some patients with diplopia in Graves' orbitopathy. Methods: 20 patients, aged between 20 and 80 years, of both sexes, designated as benign (eyelid retraction), intermediate(diplopia in primary sight position) and malignant or infiltrative(signs of optic nerve compromise) forms were evaluated by orbital tomography. All patients had already been euthyroidean for at least one year. Tomographic patterns were studied and divided into two groups: with or without extraocular muscle tendons enlargment. Results: Statistically significant relationship was found between tendon enlargment and intermediate form $(\mathrm{p}<0.012)$. Conclusion: Extraocular tendon involvement present in $30 \%$ of the patients with Graves' ophthalmopathy, and is positively correlated to intermediary form of the disease, characterized by diplopia, a very important clinical landmark.
\end{abstract}

Keywords: Graves, ophthalmopathy/diagnosis; Tomography, x-ray computed; Exophthalmos; Orbit

\section{INTRODUÇãO}

$\Delta$ oftalmopatia de Graves é de longe a etiologia mais frequente de proptoses uni ou bilaterais. Os cientistas têm estudado esta enfermidade desde que foi descrita na Inglaterra por Graves, em 1835, e na Alemanha por Basedow, em $1840^{(1-2)}$. As provas de função tireoideana, os títulos de anticorpos específicos ou a presença de antígenos de histocompatibilidade não se correlacionam necessariamente com o diagnóstico ou a severidade da enfermidade ocular ${ }^{(3-6)}$.

Sabe-se que as manifestações oculares da enfermidade de Graves podem ser classificadas de uma maneira muito simples em: exoftalmo benigno (ou não infiltrativo) e proptose maligna (ou infiltrativa). Existe ainda uma forma intermediária desta enfermidade na qual encontramos infiltração isolada e mais precoce e discreta dos músculos, caracterizada pela presença de estrabismo e diplopia. Esta subdivisão provem de uma simplificação do clássico mneumônico NOSPECS, criado por Werner, assim resumido em seus traços gerais ${ }^{(7,8)}$ :

Classe 0: "No signs or symptoms"

Classe 1: "Only signs,no symptoms"

Classe 2: "Soft tissue involvement"

Classe 3: "Proptosis"

Classe 4: "Extraocular muscle involvement"

Classe 5: "Corneal involvement"

Classe 6: "Sight loss"

Assim, de maneira simplificada, o exoftalmo benigno corresponde às classes $1,2 \mathrm{e} 3$, a forma intermediária corresponde à classe 4 , e a infiltrativa e a forma maligna ou infiltrativa corresponde às classes 5 e $6^{(7-9)}$.
Durante muito tempo assumiu-se que o espessamento da musculatura ocular sempre poupava os tendões extraoculares. Não obstante, os estudos de Ben Simon e colaboradores observaram o espessamento dos tendões, contestando as opiniões pré-existentes ${ }^{(10,11)}$.

Os avanços nas técnicas radiológicas e a atenção dirigida aos achados nestes últimos anos podem ser úteis naqueles casos de diagnóstico difícil, bem como no esclarecimento definitivo da sua etiopatogenia, tanto que Zonneveld et al. classificaram os casos examinados através de tomografia computadorizada em 4 grupos. No primeiro não há aumento do volume dos tecidos gorduroso ou muscular.No segundo evidencia-se apenas o aumento do volume gorduroso ${ }^{(12)}$. No terceiro, o acometimento muscular é isolado e no quarto ambos os tecidos estão afetados. Concluíram que se estes grupos são identificados é provável que envolvam mecanismos etiopatogênicos distintos e que necessitem abordagens terapêuticas diferenciadas. ${ }^{12-20}$

Muitos autores tem estudado as medidas dos tecidos orbitários nos exames de imagem ${ }^{(18,21,22)}$.

Estes dados nos estimularam estudar a frequência do espessamento do tendão muscular em cortes axiais das tomografias computadorizadas de órbita, realizadas em pacientes com oftalmopatia de Graves, buscando estabelecer correlações com as diferentes formas clínicas da doença na oftalmopatia endócrina.

\section{Métodos}

Foi realizada tomografia computadorizada de órbita, com o scanner GE HI_SPEED CT/i de General 
Electric Systems, em cortes sagitais para analisar 20 pacientes com oftalmopatia de Graves de ambos os sexos, previamente eutiroideanos por pelo menos um ano, com seguimento no Serviço de órbita do Instituto Penido Burnier na cidade de Campinas, São Paulo, Brasil.

A apresentação clínica foi estruturada com base na tradicional classificação conhecida como NOSPECS ${ }^{(7-9)}$ :

Aqueles pacientes sem sinais ou sintomas (classe $0)$ não foram estudados.

1. Oftalmopatia benigna (classes de 1, 2 e 3): pacientes com retração palpebral.

2. Oftalmopatia intermediária (classe 4): pacientes com estrabismo e diplopia na posição primária do olhar.

3. Oftalmopatia infiltrativa (maligna) (classes 5 e 6): pacientes com exposição corneana ou sinais de neuropatia óptica.

Todos os pacientes foram examinados por um único médico oftalmologista.

O espessamento do tendão muscular foi procurado nos retos mediais e laterais, melhor visualizados em cortes sagitais (axiais).
As medidas dos ventres musculares foram consideradas aumentadas se ultrapassassem os valores normais encontrados em estudo realizado por Zonneveld et al., após tomografia computadorizada em 40 órbitas, conforme descritos a seguir ${ }^{(12)}$ :

Músculo reto mediano - $4.1 \pm 0.5 \mathrm{~mm}$ de espessura no ponto médio da órbita;

Músculo reto lateral - $2.9 \pm 0.6 \mathrm{~mm}$ de espessura no ponto médio da órbita;

Nervo óptico - $4.2 \pm 0.6 \mathrm{~mm}$ de espessura no ponto médio da órbita.

As medidas dos tendões no equador do globo foram consideradas normais se correspondiam a, no máximo, metade da medida dos ventres musculares ${ }^{(18)}$.

Por último foram calculadas as relações entre a medida do tendão e do ventre muscular, objeto deste estudo.

Os achados tomográficos foram classificados em dois grupos, de acordo com a observação em cortes axiais:

1. Com espessamento do tendão muscular:

Quando a relação entre a medida do tendão (no equador do globo) e do ventre muscular (no ponto médio da órbita) foi igual ou superior a $0,5^{(10)}$. (Figuras 1 e 2).

Tabela 1

Relação dos pacientes estudados através de tomografia computadorizada em busca do espessamento dos tendões

\begin{tabular}{|c|c|c|c|c|c|c|c|c|}
\hline \multirow[t]{2}{*}{ Nome } & \multirow[t]{2}{*}{$N^{0}$ da ficha } & \multirow[t]{2}{*}{ Idade } & \multirow[t]{2}{*}{ Sexo } & \multicolumn{3}{|c|}{ Forma clínica } & \multicolumn{2}{|c|}{ Espessamento dos tendões } \\
\hline & & & & $\mathbf{B}$ & $\mathbf{I}$ & $\mathbf{M}$ & Sem espessamento & Com espessamento \\
\hline GR & 1107082 & 22 & $\mathrm{~F}$ & & $X$ & & & $\mathrm{X}$ \\
\hline MAS & 905316 & 55 & $\mathrm{~F}$ & & $\mathrm{X}$ & & & $\mathrm{X}$ \\
\hline OSP & 967075 & 58 & $\mathrm{~F}$ & & $\mathrm{X}$ & & $\mathrm{X}$ & \\
\hline $\mathrm{NC}$ & 1054642 & 58 & $\mathrm{~F}$ & & & $\mathrm{X}$ & $\mathrm{X}$ & \\
\hline IAN & 1026260 & 36 & $\mathrm{~F}$ & & $\mathrm{X}$ & & $\mathrm{X}$ & \\
\hline $\mathrm{EF}$ & 1103446 & 33 & $\mathrm{M}$ & & $\mathrm{X}$ & & & $\mathrm{X}$ \\
\hline LCVC & 931684 & 55 & M & $\mathrm{X}$ & & & $\mathrm{X}$ & \\
\hline $\mathrm{DF}$ & 1025221 & 40 & $\mathrm{~F}$ & $\mathrm{X}$ & & & & \\
\hline $\mathrm{JO}$ & 983283 & 29 & $\mathrm{~F}$ & & & $X$ & $X$ & \\
\hline AOAP & 1118859 & 74 & $\mathrm{~F}$ & & $X$ & & $X$ & \\
\hline IMFT & 1064519 & 48 & $\mathrm{~F}$ & $X$ & & & & \\
\hline MMC & 1101022 & 45 & $\mathrm{~F}$ & $X$ & & & & \\
\hline RSV & 1098380 & 46 & $\mathrm{~F}$ & & $X$ & & & $\mathrm{X}$ \\
\hline LAS & 1110460 & 27 & $\mathrm{~F}$ & $\mathrm{X}$ & & & & \\
\hline MRZL & 763239 & 38 & M & $\mathrm{X}$ & & & & \\
\hline $\mathrm{DB}$ & 1080046 & 43 & M & & $X$ & & & $X$ \\
\hline $\mathrm{CB}$ & 983482 & 51 & M & & $X$ & & & $X$ \\
\hline AGL & 1112775 & 56 & $\mathrm{~F}$ & & $X$ & & $X$ & \\
\hline JRRR & 1122784 & 55 & M & & & $\mathrm{X}$ & & \\
\hline MJM & 1068622 & 67 & $\mathrm{~F}$ & & & $X$ & $X$ & \\
\hline
\end{tabular}

B - benigno; I - intermediária; M - infiltrativa; 6 casos de espessamento são do reto mediano 
2. Sem espessamento do tendão muscular: quando a relação entre a medida do tendão no equador do globo ocular e do ventre muscular no ponto médio da órbita foi menor que 0,5 . Neste grupo foram incluídos os casos em que foi observado apenas o espessamento do ventre muscular (posterior) ou dos tecidos conectivoadiposo.

As medidas foram feitas por um único aparelho de tomografia, e por um único médico radiologista, com interesse especial em patologias orbitárias e que desconhecia os achados clínicos de cada paciente.

\section{Resultados}

Dos 20 pacientes estudados, 14 eram do sexo feminino e 6 do sexo masculino; 6 apresentavam a forma clínica benigna, 10 a intermediária e 4 a forma maligna da oftalmopatia de Graves.

Os resultados foram analisados de acordo com teste do Qui-Quadrado (Exato de Fischer) na análise de associação e o teste de Kappa na avaliação de concordância.

Encontramos uma associação estatisticamente significativa entre espessamento sagital dos tendões e a forma clínica intermediária $(\mathrm{p}<0,012)$. Como encontramos uma significância estatística, sendo esta a variável mais importante neste estudo, pode-se concluir que tamanho da amostra, neste caso, está adequado.

No grupo sem espessamento dos tendões não havia forma clínica predominante $(4,29 \%$ apresentavam a forma benigna, $26 \%$ a intermediária e $28.6 \%$ apresentavam oftalmopatia infiltrativa).

No grupo com espessamento do tendão, todos os pacientes apresentavam oftalmopatia intermediária e em todos o músculo espessado foi o reto mediano. Nenhum deles apresentou espessamento do reto lateral. Quatro pacientes com forma intermediária não apresentaram espessamento do tendão em suas tomografias (Tabela 1).

\section{Dıscussão}

Como mencionamos antes, não existem provas sorológicas que permitam confirmar o diagnóstico de oftalmopatia endócrina ${ }^{(13,23)}$.

A ecografia ocular pode ser fácil e rapidamente realizada, e por este, motivo não podemos desvalorizar sua indicação ${ }^{(24)}$.

Com a ressonância nuclear magnética, ainda não acessível a todos os pacientes em nosso meio, podería-

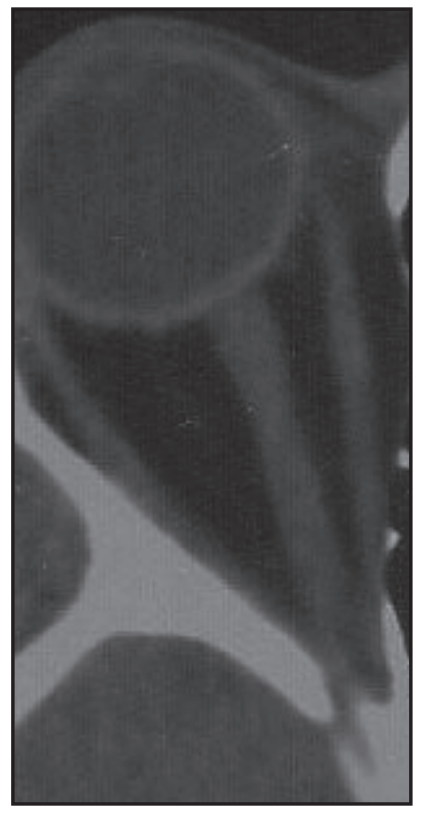

Figura 1 - Aspecto muscular em uma órbita normal. Notar a medida do ventre e do tendão muscular em relação ao nervo óptico

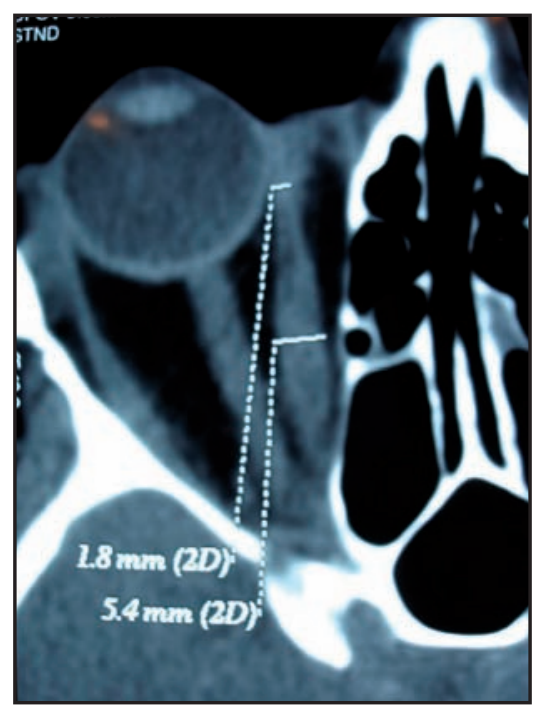

Figura 2: Medidas do tendão no equador do globo e do ventre muscular no ponto médio da órbita; Nota-se que quando a relação entre as medidas do tendão e do ventre muscular é menor que 0,5 o padrão é fusiforme

mos estudar inclusive o conteúdo líquido dos tecidos, que reflete edema, e deste modo, a atividade inflamatória da enfermidade orbitária ${ }^{(25)}$.

A facilidade de realização do exame por um mesmo serviço de radiologia da nossa comunidade nos levou a optar pela tomografia computadorizada. A despeito das possibilidades existentes à ressonância magnética já mencionadas, a tomografia é muito eficiente na avaliação dos tecidos orbitários porque mostra a diferença de contraste entre diversas estruturas: gordura, músculos, fluídos intraoculares, seios paranasais e ossos, e,portanto, bastante eficaz, tendo em vista o objetivo deste trabalho ${ }^{(15)}$.

A forma benigna aparece na etapa de 


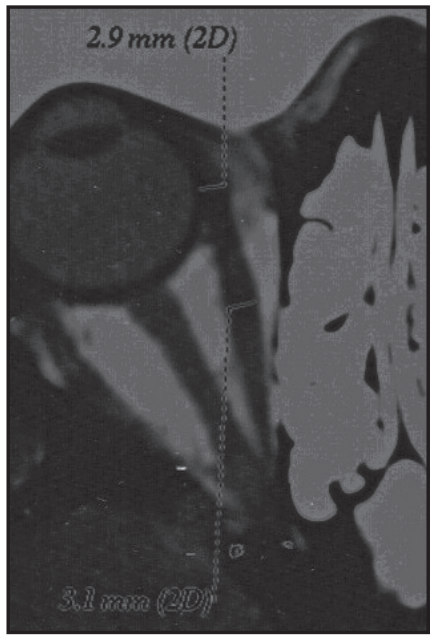

Figura 3: Medidas do tendão no equador do globo e do ventre muscular no ponto médio da órbita; Notar o aspecto cilíndrico do reto mediano hipertireoidismo e é causada por um estado de hipertônus simpático, que estimula o músculo de Müller e os retratores da pálpebra inferior, causando exposição corneana e "scleral show" com pseudoproptose. Alguns casos não regridem espontaneamente quando os níveis de hormônios tiroideanos retornam à normalidade, por fibrose destes músculos. Os achados radiológicos neste estudo limitaram-se ao aumento dos tecidos conectivoadiposo, sem espessamento muscular evidente. Este fato apóia as hipóteses sugeridas por Char (1990) de que o comprometimento orbitário se manifesta afetando o tecido conectivo-adiposo ou o muscular de diferentes maneiras. É provável que os pacientes com hipertrofia de tecido gorduroso não manifestem sinais de inflamação da musculatura extraocular. No presente estudo, o espessamento muscular sem comprometer os tendões foi observado em apenas um caso, levando-nos a aceitar o que fora anteriormente mencionado pelos autores (9,18,25,26) (Figura 1) .

A forma intermediária deve ser considerada em pacientes que apresentam estrabismo com diplopia na posição primária do olhar, especialmente se o paciente afetado for homem ou mulher após a menopausa. $\mathrm{O}$ desequilíbrio muscular é tipicamente intermitente e geralmente vertical, de predomínio matutino pelo edema orbitário máximo neste período do dia. No presente estudo, 7 mulheres e 3 homens apresentaram esta forma clínica.Todos estavam entre a terceira e quarta décadas de vida. Neste estudo três pacientes que apresentavam a forma intermediária da oftalmopatia de Graves, apresentavam espessamento muscular posterior discreto sem espessamento do tendão. $\mathrm{O}$ achado mais frequente neste grupo foi o espessamento muscular anterior, afetando os tendões, apoiando a hipótese formulada por Ben Simon. Neste estudo há uma correlação

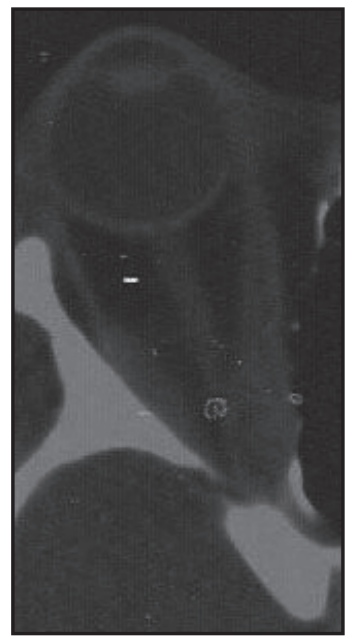

Figura 4: Aspecto do espessamento muscular mais posterior, levando à compressão do nervo óptico no ápice da órbita, especialmente pelo reto mediano estatisticamente significativa entre este achado e a forma clínica intermediária $(\mathrm{p}<0,012)^{(6,11)}$.

O espessamento muscular quando a relação entre as medidas do tendão e do ventre muscular foi maior ou igual a 0,5 foi discreto e o aspecto lembra mais um padrão cilíndrico do que o espessamento fusiforme observado nos casos mais graves da doença ${ }^{(27)}$.

Em cortes axiais os músculos mais facilmente estudados são os retos horizontais. No entanto, sabe-se que o estrabismo na oftalmopatia de Graves é predominantemente vertical e que o músculo mais usualmente afetado é o reto inferior, seguido pelos retos medial, superior e lateral respectivamente. O interessante é notarmos que apesar de 8 pacientes nesta série apresentarem estrabismo e diplopia caracteristicamente verticais, observou-se espessamento do tendão do reto mediano nos cortes axiais. É provável que a observação adequada do tendão do reto inferior demonstrasse também o seu espessamento, talvez em cortes sagitais. Outros estudos poderiam comprovar esta hipótese ${ }^{(25)}$.

O espessamento muscular (quando a relação entre as medidas do tendão e do ventre muscular foi maior ou igual a 0,5 ) foi discreto e o aspecto lembra mais um padrão cilíndrico do que o espessamento fusiforme observado nos casos mais graves da doença ${ }^{(27)}$ (Figuras 2 e 3 ).

Vários estudos sugerem que a severidade da proptose se relaciona com o aumento do tecido adiposo, mais do que com o espessamento muscular, diferindo do que encontramos neste estudo. Pelo contrário, acreditamos que a expansão dos ventres musculares, posteriormente poderia facilitar a ectasia venosa no ápice da órbita, perpetuando um ciclo vicioso de proptose e congestão, levando inexoravelmente ao glaucoma póstrabecular, a neuropatia óptica por compressão e finalmente à perda da visão ${ }^{(26,28)}$ (Figura 4). 
Barret et al. descreveram um método bastante reprodutível para correlacionar o grau de espessamento da musculatura extrínseca e a possibilidade de se desenvolver neuropatia óptica. Segundo os autores a porcentagem do volume orbitário ocupado pelos músculos retos horizontais e verticais no ponto médio da órbita maior ou igual a $67 \%$ é dado praticamente diagnóstico para neuropatia óptica nesta patologia ${ }^{(24)}$.

Assim como o índice de Barret se mostra bastante expressivo nos casos de neuropatia na oftalmopatia de Graves, o achado de espessamento dos tendões parece ser fortemente indicativo de estrabismo nesta pa$\operatorname{tologia}^{(24)}$.

\section{Conclusão}

O achado tomográfico de espessamento dos tendões está presente em $30 \%$ dos pacientes com diagnóstico clínico de oftalmopatia de Graves, relaciona-se positivamente com a forma intermediária da doença, caracterizada pela presença de diplopia, e que constitui um achado de grande valor clínico.

\section{ReferêNCIAS}

1. Graves RJ. A newly observed affection of the thyroid gland in females. London Med Surg J. 1835;7(173):516-7.

2. Frecker M, Stenszky V, Balazs C, Kozma L, Kraszits E, Farid NR. Genetic factors in Graves' ophthalmopathy. Clin Endocrinol (Oxford). 1986;25(5):479-85.

3. Naffziger HC, Jones OW Jr. The surgical treatment of progressive exophthalmos following thyroidectomy. JAMA 1932;99(8):638-42.

4. Kriss JP, Pleshakov V, Rosenblum AL, Holderness M, Sharp G Utiger R. Studies on the pathogenesis of the ophthalmopathy of Graves' disease. J Clin Endocrinol Metab. 1967;27(4):582-93.

5. Hetzel BS, Mason EK, Wang HK. Studies of serum longacting thyroid stimulator (LATS) in relation to exophthalmos after therapy for thyrotoxicosis. Australas Ann Med. 1968;17(4):307-11.

6. Abreu JMQ, Abreu M. Oftalmopatia hipófiso-tireoideana. Arq Inst Penido Burnier. 1961;18:76-93.

7. Werner SC. A new classification of the eye changes of Graves' disease. Arch Ophthalmol. 1969;82(3):421-3.

8. Abreu EB, Lopes LCF, Federmann I. Oftalmopatia de Graves: revisão e atualização. Arq Inst Penido Burnier. 1994;36(1):32-41.

9. Ben Simon GJ, Syed HM, Douglas R, McCann JD, Goldberg RA. Extraocular muscle enlargement with tendon involvement in thyroid-associated orbitopathy. Am J Ophthalmol. 2004;137(6):1145-7.

10. Szucs-Farkas Z, Toth J, Balazs E, Galuska L, Burman KD, Karanyi Z, et al. Using morphologic parameters of extraocular muscles for diagnosis and follow-up of Graves' ophthalmopathy: diameters, areas, or volumes? AJR Am J Roentgenol. 2002;179(4):1005-10.
11. Zonneveld FW, Koornneef L, Wittebol-Post D. Quantitative volumetric assessment of orbital soft tissue. In: von Lemke HU, Rhodes ML, Jaffe CC, Felix R, editors. Computer assisted radiology: proceedings of the International Symposium CAR' 91. Berlin: Springer-Verlag; 1991. p. 181-6.

12. Weber AL, Dallow RL, Sabates NR. Graves' disease of the orbit. Neuroimaging Clin N Am. 1996;6(1):61-72

13. Barthey GB, Gorman CA. Diagnostic criteria for Graves' ophthalmopathy. Am J Ophthalmol. 1995;119(6):792-5.

14. Forbes G, Gorman CA, Brennan MD, Gehring DG, IIlstrup DM, Earnest F 4th. Ophthalmopathy of Graves' disease: computerized volume measurements of the orbital fat and muscle. AJNR Am J Neuroradiol. 1986;7(4):651-6.

15. Rothfus WE, Curtin HD. Extraocular muscle enlargement: a CT review. Radiology. 1984;151(3):677-81.

16. Goodall KL, Jackson A, Leatherbarrow B, Whitehouse RW. Enlargement of the tensor intermuscularis muscle in Graves' ophthalmopathy. A computed tomographic and magnetic resonance imaging study. Arch Ophthalmol. 1995;113(10):1286-9

17. Nugent RA, Belkin RI, Neigel JM, Rootman J, Robertson WD, Spinelli J, Graeb DA. Graves's orbitopathy: correlation of CT and clinical findings. Radiology. 1990;177(3):675-82.

18. Trokel SL, Hilal SK. Submillimeter resolution CT scanning of orbital diseases. Ophthalmology. 1980;87(5):412-7

19. Kennerdell JS, Rosenbaum AE, El-Hoshy MH. Apical optic nerve compression of dysthyroid optic neuropathy on computed tomography. Arch Ophthalmol. 1981;99(5):807-9.

20. Regensburg NI, Kok PH, Zonneveld FW, Baldeschi L, Saeed P, Wiersinga WM, Mourits MP. A new and validated CT-based method for the calculation of orbital soft tissue volumes. Invest Ophthalmol Vis Sci. 2008;49(5):1758-62.

21. Regensburg NI, Wiersinga WM, van Velthoven ME, Berendschot TT, Zonneveld FW, Baldeschi L, et al. Age and gender-specific reference values of orbital fat and muscle volumes in Caucasians. Br J Ophthalmol. 2010 Jun 7. [Epub ahead of print].

22. Takeda Y, Kriss JP. Radiometric measurement of thyroglobulin- antithyroglobulin immune complex in human serum. J Clin Endocrinol Metab. 1977:44(1):46-55.

23. Monteiro ML, Gonçalves AC, Silva CT, Moura JP, Ribeiro CS, Gebrim EM. Diagnostic ability of Barrett's index to detect dysthyroid optic neuropathy using multidetector computed tomography. Clinics (São Paulo). 2008;63(3):301-6.

24. Machado KFS, Garcia MM. Oftalmopatia tireoidea revisitada. Radiol Bras. 2009;42(4):261-6

25. Patrinely JR, Osborn AG, Anderson RL, Whiting AS. Computed tomographic features of nonthyroid extraocular muscle enlargement. Ophthalmology. 1989;96(7):1038-47. Comment in Ophthalmology. 1990;97(1):4-5.

26. Garrity JA, Bahn RS. Pathogenesis of graves ophthalmopathy: implications for prediction, prevention, and treatment. Am J Ophthalmol. 2006;142(1):147-53.

27. Rubin PA, Watkins LM, Rumelt S, Sutula FC, Dallow RL. Orbital computed tomographic characteristics of globe subluxation in thyroid orbitopathy. Ophthalmology. 1998;105(11):2061-4.

28. Regensburg NI, Wiersinga WM, Berendschot TT, Potgieser P, Mourits MP. Do subtypes of graves' orbitopathy exist? Ophthalmology. 2011;118(1):191-6.

\author{
Endereço para Correspondência: \\ Elvira Barbosa Abreu \\ Instituto Penido Burnier \\ Rua Dr. Mascarenhas, $n^{\circ} 249$ \\ CEP 13013-175 - Campinas (SP), Brasil \\ E-mail: barbosaabreu.oftalmo@terra.com.br
}

